\title{
VISUAL EVOKED POTENTIALS IN PHENYLKETONURIA
}

Visual evoked potentials (VEPs) were studied in 36 patients with phenylketonuria, and compared with MRI findings, dietary state, and IQ at the Institute of Neurology, Queen Square, and the Institute of Child Health, Great Ormond Street Hospital, London, UK. Four patients presented with progressive neurological deficits. Twenty six were detected by routine neonatal screening, and 9 were diagnosed with developmental delay at age 5 years. All except one showing no symptoms had received a low phenylalanine diet until age 7 years or later. In 9 patients aged less than 14 years who were still on the diet, VEPs were normal in 8 and the MRI was abnormal in all but only mildly irregular in 6 . The one patient with abnormal VEPs had the most pronounced MRI abnormalities and the lowest IQ score (74 compared to 90-119 in the remaining 8 ). Of 27 patients aged 14-31 years, $>80 \%$ had abnormal VEPs, showing reduction of amplitude and prolonged latencies, whereas neuro-ophthalmological examination was normal. VEP amplitude was inversely correlated with MRI abnormalities and severity of white matter lesions in the parieto-occipital region, and with IQ. VEP abnormalities were marginally correlated with plasma phenylalanine concentrations but were not dependent on a sustained low phenylalanine diet. (Jones SJ et al. Visual evoked potentials in phenylketonuria: association with brain MRI, dietary state, and IQ. I Neurol Neurosurg Psychiatry September 1995;59:260-265). (Respond: Dr SJ Jones, Department of Clinical Neurophysiology, National Hospital for Neurology and Neurosurgery, Queen Square, London WC1N 2BG, UK).

COMMENT. This study confirms reports of the frequency of abnormal VEPs in patients with phenylketonuria, even in those diagnosed and treated early. VEP decreased amplitude and prolonged latency are correlated to some extent with subcortical myelin defects revealed by MRI. A striking increase in incidence of VEP and MRI abnormalities in older patients coincided with relaxed dietary management, plasma phenylalanine concentrations, and intellectual performance.

\section{TOXIC DISORDERS}

\section{MRI IN KERNICTERUS}

The magnetic resonance images (MRI) of three children with athetotic cerebral palsy and severe neonatal jaundice were examined in the Department of Pediatric Neurology, Ohzora-no-iye Hospital and Seirei-Mikatahara General Hospital, Shizuoka, Japan. High intensity areas in the posteromedial border of the globus pallidus on T2-weighted images were found bilaterally in all 3 children. No abnormalities were demonstrated on T1-weighted imaging. (Yokochi K. Magnetic resonance imaging in children with kernicterus. Acta Paediatr August 1995;84:937-9). (Respond: Dr K Yokochi, Ohzora-no-iye Hospital, $\overline{7448}$ Nakagawa, Hosoe, Inasa, Shizuoka 431-13, Japan).

COMMENT. Kernicteric encephalopathy is a rare neonatal disorder since the introduction of phototherapy. Autopsy findings have revealed bilirubin staining of the globus pallidus, subthalamic nucleus, hippocampus, and dentate and olivary nuclei. The posteromedial border of the globus pallidus is the most sensitive region to kernicterus in MR imaging. Perinatal hypoxic-ischemic encephalopathy is distinguished by involvement of the putamen and thalamus on pathological and MR 